\title{
Characterization of the In Vitro Inhibitory Potential of the Oligonucleotide Imetelstat on Human Cytochrome P450 Enzymes with Predictions of In Vivo Drug-Drug Interactions ${ }^{\text {ฐ }}$
}

\author{
(1) Faraz Kazmi, Carlo Sensenhauser, and Tony Greway \\ Drug Metabolism and Pharmacokinetics, Janssen Research \& Development, LLC, Spring House, Pennsylvania (F.K., C.S.) and \\ Drug Metabolism and Pharmacokinetics, Janssen Research \& Development, LLC., Raritan, New Jersey (T.G.)
}

Received August 28, 2018; accepted October 29, 2018

\begin{abstract}
Imetelstat, a 13-base oligonucleotide (5'-TAGGGTTAGACAA-3'), is a potent, investigational telomerase inhibitor in clinical development for the treatment of hematologic myeloid malignancies. Modifications to imetelstat oligonucleotide chemistry include an N3'-P5' thio-phosphoramidate backbone linkage to improve biologic stability and the addition of a palmitoyl tail at the $5^{\prime}$-position to enhance cellular membrane permeability. Other oligonucleotides have been previously shown to have in vitro test-system-dependent outcomes when potent cytochrome $\mathbf{P 4 5 0}$ inhibition in human liver microsomes (HLM) is observed, but such inhibition is not observed in cryopreserved human hepatocytes (CHH). Outcomes in $\mathrm{CHH}$ are consistent with clinical reports in which no interactions were reported. In the present study, imetelstat was evaluated for in vitro inhibition of eight P450 enzymes, namely CYP1A2, CYP2B6, CYP2C8, CYP2C9,
\end{abstract}

CYP2C19, CYP2D6, CYP2E1, and CYP3A4 in CHH (0.5 million cells $/ \mathrm{ml})$. Assays were performed using validated conditions, including short substrate times (10 minutes), and at the approximate substrate $K_{m}$ concentration. Imetelstat was found to have little to no inhibition of all P450 isoforms evaluated, with inhibitor concentration that causes $50 \%$ inhibition $\left(\mathrm{IC}_{50}\right)$ values $>100 \mu \mathrm{M}$. Maximum percent inhibition values for each P450 isoform at $100 \mu \mathrm{M}$ imetelstat were $<20 \%$ except for CYP2C8 activity, which was inhibited by $49 \%$. Using a static mechanistic model, the predicted change in area under the curve of a victim drug coadministered with imetelstat was 1.04-fold, projecting no relevant clinical interaction. Overall, the results from this in vitro study suggest that clinical use of imetelstat is unlikely to affect the pharmacokinetics of concomitant therapies that undergo cytochrome P450-mediated metabolism.

\section{Introduction}

Oligonucleotide based therapeutics are an attractive area for pharmaceutical development due to the ability to provide target specificity with base-pair sequence, especially in the areas of cancer treatment, genetic disorders, infectious diseases, and metabolic disorders (Lundin et al., 2015; Sharma and Watts, 2015; Aartsma-Rus, 2016). Usually, these molecules function by having an anti-sense base-pair sequence complementary to a target mRNA sequence, leading to downregulation of mRNA translation and protein expression, and are thus also known as anti-sense oligonucleotides (ASOs). Chemical modification of oligonucleotides has been adopted as a strategy to enhance target interaction and biologic stability, yielding a general improvement in pharmacokinetic and pharmacodynamic properties of these therapeutics. This includes sulfurization of the phosphodiester bond, methoxy or methoxyethyl group addition to sugar moieties, locked/unlocked nucleic acids, and internucleotide linkage modifications to form peptide nucleic acids (Prakash, 2011; Deleavey and Damha, 2012; Sharma and Watts, 2015; Chen et al., 2018). Among the many oligonucleotide biomolecules investigated thus far, only fomivirsen, pegaptanib, mipomersen, eteplirsen, nusinersen, and

https://doi.org/10.1124/dmd.118.084103.

S This article has supplemental material available at dmd.aspetjournals.org. defibrotide sodium have received U.S. Food and Drug Administration (FDA) approval (Stein and Castanotto, 2017).

Imetelstat (13-mer oligonucleotide N3'-P5' thio-phosphoramidate with a covalently linked C16 [palmitoyl] lipid moiety at the $5^{\prime}$ end) is in clinical development as a treatment option for hematologic myeloid malignancies (Röth et al., 2010; Baerlocher et al., 2015; Chiappori et al., 2015; Tefferi et al., 2015; Dillen et al., 2017). Imetelstat is a highaffinity, active-site telomerase inhibitor with specificity for the RNA template region of telomerase, directly competing with telomere binding. Therefore, its mode of action is similar to a conventional small-molecule competitive enzyme inhibitor rather than like an ASO (knockdown of target) (Herbert et al., 2005). Activation of telomerase is thought to be important for tumor progression by enabling cancer cells to maintain short telomeres, gain immortality, and evade apoptosis (Lichtsteiner et al., 1999). The mechanism of action by imetelstat results in potent inhibition of telomerase and leads to progressive telomere shortening (Herbert et al., 2005; Marian et al., 2010). Structurally (see Fig. 1), the thio-phosphoramidate linkage within imetelstat confers high resistance to nuclease digestion in blood and tissues, while the properties of the $5^{\prime}$-palmitoyl chain result in improved cellular permeability and consequently strong tissue penetration and retention (Herbert et al., 2005).

The potential for oligonucleotide-based molecules to act as perpetrators of drug-drug interactions (DDIs) has not been reported extensively in the literature. However, of the clinical interaction studies conducted

ABBREVIATIONS: ASO, anti-sense oligonucleotides; AUC, area under the curve; $\mathrm{CHH}$, cryopreserved human hepatocytes; DDI, drug-drug interaction; EMA, European Medicines Agency; FDA, U.S. Food and Drug Administration; HLM, human liver microsomes; IC 50 , inhibitor concentration that causes $50 \%$ inhibition; KHB, Krebs-Henseleit buffer; P450, cytochrome P450; TDI, time-dependent inhibition. 


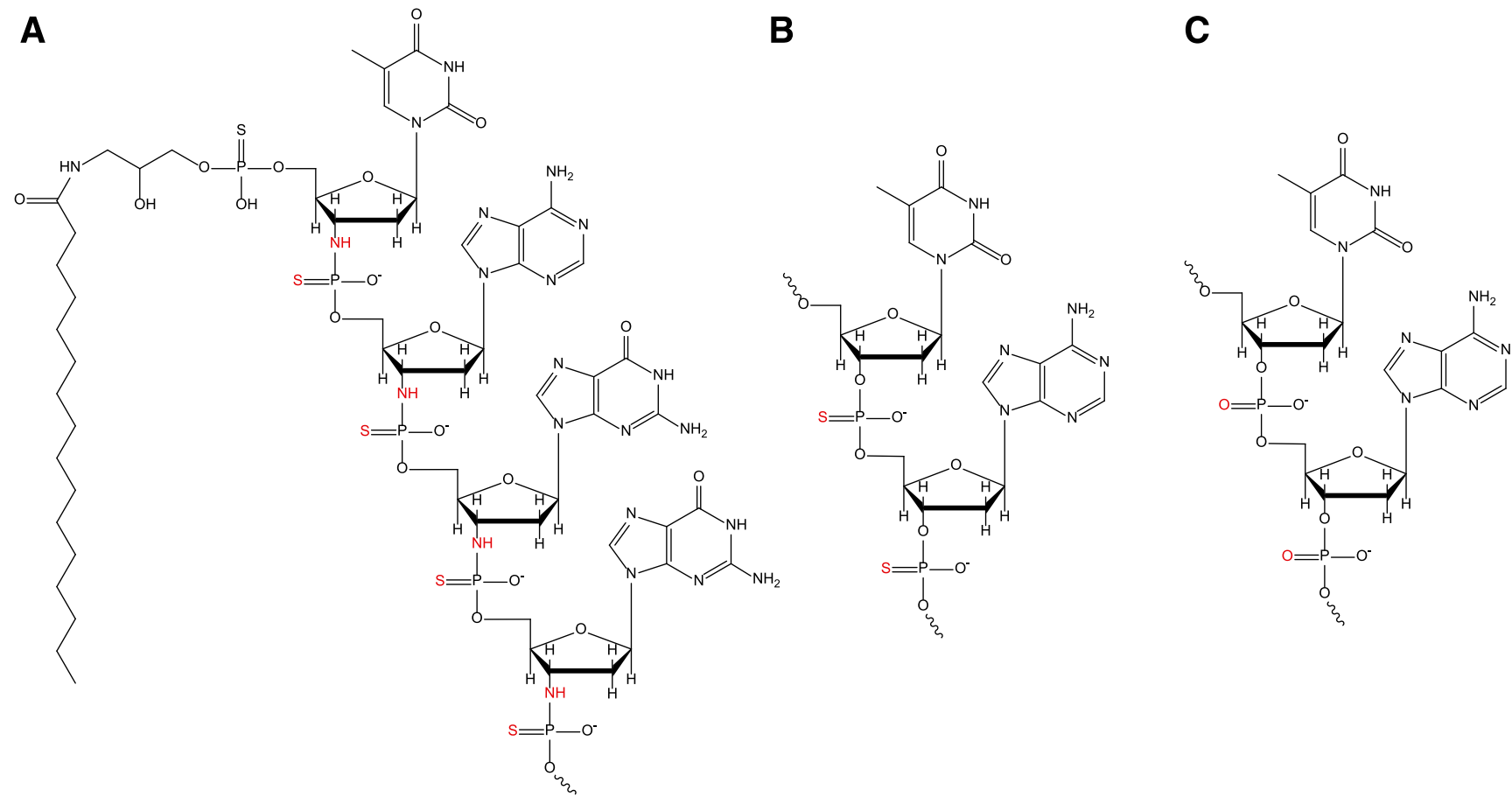

Fig. 1. Truncated structures of (A) imetelstat, (B) phosphorothioate, and (C) phosphodiester oligonucleotide linkages. The thio-phosphoramidate, phosphorothioate, and phosphodiester linkages on each respective molecule are highlighted in red.

with victim drugs subject to clearance by cytochrome P450, glucuronidation, renal elimination, or nucleoside kinases, none has reported a clinically relevant DDI by therapeutic oligonucleotides (Adjei et al., 2003; Villalona-Calero et al., 2004; Geary et al., 2006; Yu et al., 2009; Li et al., 2014). Industry regulatory guidances from the FDA (2017 draft-https://www.fda.gov/downloads/Drugs/ GuidanceComplianceRegulatoryInformation/Guidances/UCM581965.pdf) and the European Medicines Agency (EMA; 2013 final- http://www. ema.europa.eu/docs/en_GB/document_library/Scientific_guideline/2012/07/ WC500129606.pdf) recommend in vitro P450 inhibition studies for small-molecule drugs in appropriate test systems (such as human liver microsomes or cryopreserved human hepatocytes) to ascertain the perpetrator DDI potential of a drug. Recent studies on P450 inhibition in vitro suggest that cryopreserved human hepatocytes $(\mathrm{CHH})$ are a more clinically relevant test system for the prediction of DDIs by oligonucleotides, and that studies conducted in human liver microsomes may result in artifactual results (Kazmi et al., 2018). This is supported by another recent in vitro report in which no P450 inhibition by oligonucleotides in $\mathrm{CHH}$ was reported (Shemesh et al., 2017). In the present study, the in vitro P450 inhibitory potential of imetelstat was evaluated in $\mathrm{CHH}$ to determine the risk for $\mathrm{P} 450$ mediated DDIs with concomitantly administered medications.

\section{Materials and Methods}

Chemicals and Reagents. Imetelstat, a 13-base oligonucleotide (5'-TAGGGTTAGACAA-3') N3'-P5' thio-phosphoramidate containing a palmitoyl tail at the $5^{\prime}$-position ( $5^{\prime}$-palmitoyl-TAGGGTTAGACAA-NH2-3') was provided by Geron Corporation (Menlo Park, CA). Krebs-Henseleit buffer (KHB), calcium chloride, phenacetin, bupropion, tolbutamide, dextromethorphan, chlorzoxazone, midazolam, $\alpha$-naphthoflavone, ticlopidine, sulfaphenazole, quinidine, 4-methylpyrazole, ketoconazole, amodiaquine, montelukast, and acetaminophen were all purchased from MilliporeSigma (St. Louis, MO). Sodium bicarbonate and HEPES were purchased from Life Technologies/Thermo Fisher Scientific (Carlsbad, CA). $N$-3-benzylphenobarbital was obtained internally from Janssen Research
\& Development (Beerse, Belgium); $S$-(+)-mephenytoin was purchased from Corning Life Sciences (Corning, NY); metabolite reference standards including hydroxytolbutamide, $(+/-)-4$ '-hydroxymephenytoin, dextrorphan, 6-hydroxychlorzoxazone, 1'-hydroxymidazolam, hydroxybupropion, and $\mathrm{N}$ desethylamodiaquine were purchased from Ultrafine Chemicals and BD Gentest, both formerly part of BD Biosciences and now part of Corning Discovery Labware, Inc. (Woburn, MA). Hepatocyte Thawing Media was purchased from Bioreclamation IVT (Baltimore, MD). The Strata Impact protein precipitation 96-well plates were purchased from Phenomenex Corporation (Torrance, CA). All other reagents and solvents were of high-performance liquid chromatography grade or equivalent analytical standards.

Test Systems. Pooled cryopreserved human hepatocytes, lot PDI [cryopreserved human hepatocytes ( $\mathrm{CHH}$ ), $n=20$, mixed gender] were purchased from Bioreclamation IVT. Hepatocyte incubations were performed in oxygen-sparged KHB supplemented with calcium chloride, sodium bicarbonate and HEPES. Buffer $\mathrm{pH}$ was adjusted to $7.4 \pm 0.1$.

In Vitro P450 Inhibition. Imetelstat $(0.1-100 \mu \mathrm{M})$ was evaluated for its potential to reversibly inhibit eight $\mathrm{P} 450$ enzymes, namely CYP1A2, CYP2B6, CYP2C8, CYP2C9, CYP2C19, CYP2D6, CYP2E1, and CYP3A4 in $\mathrm{CHH}$ at the approximate substrate $\mathrm{K}_{\mathrm{m}}$ concentration (determined in house with the same lot of $\mathrm{CHH}$ ) as shown in Table 1. Triplicate incubations for each concentration were conducted with an automated liquid handling system (Biomek; Beckman Coulter, Indianapolis, IN) in 100- $\mu$ l mixtures at 0.5 million cells $/ \mathrm{ml}$ in KHB. After serial dilution in KHB, imetelstat, or experimental controls (single concentrations), and the probe substrates were aliquoted equally $(10 \mu \mathrm{leach})$ into the incubation vessels. The final organic content was $\leq 0.05 \%$ for imetelstat and $\leq 0.15 \%$ for experimental controls (with the exception of $\leq 0.85 \%$ for montelukast) in each incubated sample (considering the substrate and the inhibitor). Reactions were initiated with the addition of $80 \mu \mathrm{l} \mathrm{CHH}$ to the incubation vessels and incubations were conducted at $37^{\circ} \mathrm{C}$ with $95 \%$ humidity, $5 / 95 \% \mathrm{CO}_{2} /$ air, and $350 \mathrm{rpm}$ shaking for 10 minutes. Reactions were quenched after 10 minutes by the addition of $200 \mu \mathrm{l}$ of acetonitrile containing an appropriate mixture of internal standards. After incubation, the samples were transferred to a 2-ml Strata Impact protein precipitation 96-well plate (Phenomenex) containing $300 \mu \mathrm{l}$ of acetonitrile. The samples were filtered on a vacuum manifold, the filtrate was evaporated to dryness under nitrogen, and then reconstituted in $250 \mu 1$ mobile phase (1:1, methanol/water 
TABLE 1

Experimental conditions for the measurement of P450 activity in pooled cryopreserved human hepatocytes

\begin{tabular}{|c|c|c|c|c|c|c|c|}
\hline Enzyme & Marker Activity & $\begin{array}{c}\text { Substrate } \\
\text { Concentration }\end{array}$ & $\mathrm{CHH}$ & Incubation Time & $\begin{array}{l}\text { Mass transition } \\
\text { Monitored }\end{array}$ & Ionization Mode & Internal Standard \\
\hline & & $\mu M$ & million cells $/ \mathrm{ml}$ & $\min$ & $m / z$ & & \\
\hline CYP1A2 & Phenacetin $O$-deethylation & 20 & 0.5 & 10 & $152 / 110$ & ESI+ & $\mathrm{d}_{4}$-Acetaminophen \\
\hline CYP2B6 & Bupropion hydroxylation & 10 & 0.5 & 10 & $256 / 167$ & ESI+ & $\mathrm{d}_{6}$-Hydroxybupropion \\
\hline CYP2C8 & Amodiaquine $N$-dealkylation & 3 & 0.5 & 10 & $328 / 283$ & ESI+ & $\mathrm{d}_{3}-N$-Desethylamodiaquine \\
\hline CYP2C9 & Tolbutamide hydroxylation & 20 & 0.5 & 10 & $287 / 171$ & ESI+ & $\mathrm{d}_{9}$-Hydroxytolbutamide \\
\hline CYP2C19 & $S$-Mephenytoin $4^{\prime}$-hydroxylation & 5 & 0.5 & 10 & $235 / 150$ & ESI+ & $\mathrm{d}_{3}-4^{\prime}-$-Hydroxymephenytoin \\
\hline CYP2D6 & Dextromethorphan O-demethylation & 1 & 0.5 & 10 & $258 / 157$ & ESI+ & $\mathrm{d}_{3}$-Dextrorphan \\
\hline CYP2E1 & Chlorzoxazone 6-hydroxylation & 45 & 0.5 & 10 & $184 / 120$ & ESI- & $\mathrm{d}_{2}$-6-hydroxychlorzoxazone \\
\hline CYP3A4 & Midazolam 1'-hydroxylation & 2 & 0.5 & 10 & $342 / 324$ & ESI+ & ${ }^{13} \mathrm{C}_{3}-1^{\prime}$-Hydroxymidazolam \\
\hline
\end{tabular}

ESI, electrospray ionization.

containing $0.1 \%$ acetic acid) for further liquid chromatography/tandem mass spectrometry analysis using a cocktail method (see Table 1). Early screening studies for P450 inhibition by imetelstat in human liver microsomes (HLM) were conducted by In Vitro Technologies Inc. (Baltimore, MD) and Cerep (Redmond, WA) as described in Supplemental Table 1.

Analytical Methods. Samples were analyzed by liquid chromatography/ tandem mass spectrometry on a Shimadzu Nexera liquid chromatography system (binary LC-30AD pumps with SIL-30ACMP autosampler (Shimadzu Scientific Instruments, Columbia, MD)), coupled to a SCIEX API 4000 triplequadrupole mass spectrometer (AB SCIEX, Foster City, CA) operated in multiple reaction monitoring and electrospray ionization scan modes. For analysis of metabolites, a gradient elution method comprising $0.1 \%$ acetic acid in water (A) and methanol with $0.1 \%$ acetic acid (B) ramping from $6 \%$ to $90 \%$ over 9 minutes (vs. 4 minutes for 6-hydroxychlorzoxazone, negative electrospray ionization mode) was applied to a Zorbax Eclipse Plus Phenyl Hexyl column $(2.1 \times 100 \mathrm{~mm}, 1.8 \mu \mathrm{m}$; Agilent Technologies, Santa Clara, CA $)$ and column temperature was maintained at $50^{\circ} \mathrm{C}$.

Data Analyses. The acquired data were processed using Analyst version 1.6.2 $(\mathrm{AB}$ SCIEX) and the area ratios of metabolite and internal standard peaks were exported to Microsoft Excel 2010 (Microsoft, Redmond, WA). Nonlinear regression and inhibitor concentration that causes $50 \%$ inhibition $\left(\mathrm{IC}_{50}\right)$ curve fitting analysis were performed using SigmaPlot, version 12.5 (Systat Software, Inc., Chicago, IL). Inhibition was measured as the percent reduction of probe substrate metabolite formed in the presence of imetelstat compared with the amount formed in the absence of test compound (maximal activity). Area ratios (AR) of the metabolite peak to the internal standard peak were used in the calculations, using eq. 1 :

$$
\% \text { Inhibition }=100 \times\left[\left(\mathrm{AR}_{(\text {No Inhibitor })}-\mathrm{AR}_{(+ \text {imetelstat })}\right) / \mathrm{AR}_{(\text {No Inhibitor })}\right]
$$

Residual activity (i.e., maximal activity - \% inhibition) was plotted as a function of inhibitor concentration (numerical data in Supplemental Table 2). Prediction of hepatocyte fraction unbound $\left(\mathrm{fu}_{\mathrm{inc}}\right)$ was determined by calculation of a predicted imetelstat $\log \mathrm{P}$ with MarvinSketch version 16.1.11 (ChemAxon, Cambridge, MA) followed by application of the fu $_{\text {inc }}$ equation described previously by Kilford et al. (2008).

Imetelstat inhibition data were analyzed with basic and mechanistic static models for the assessment of DDI risk as described by the current FDA and EMA guidance documents. The static mechanistic model describing hepatic-only DDI risk is shown in eq. 2 :

$$
\text { AUC ratio }=\left(\frac{1}{[A \times B \times C] \times f_{m}+\left(1-f_{m}\right)}\right)
$$

where $\mathrm{A}=$ reversible inhibition or $\left(1 /\left(1+\left[\mathrm{I}_{\mathrm{h}}\right] / \mathrm{K}_{\mathrm{i}}\right)\right) ; \mathrm{B}=$ time-dependent inhibition (TDI) parameters; and $\mathrm{C}=$ induction parameters. Because imetelstat is dosed intravenously, gut parameters were excluded. B was assumed to equal 1 due to a lack of TDI by imetelstat (Supplemental Fig. 1). Induction parameters were not considered (i.e., $\mathrm{C}=1$ ) per the current FDA and EMA guidance recommendations on assessment of inhibition only DDI potential. $\mathrm{I}_{\mathrm{h}}=$ hepatic inlet concentration $=$ fraction unbound in plasma $\left(\mathrm{fu}_{\text {plasma }}\right) \times\left[\mathrm{C}_{\max }+\right.$ [ fraction absorbed $\left(\mathrm{F}_{\mathrm{a}}\right) \times$ absorption rate constant $\left(\mathrm{k}_{\mathrm{a}}\right) \times$ dose $\} /$ hepatic blood flow $\left.\left(\mathrm{Q}_{\mathrm{h}}\right)\right]$ /blood to plasma ratio $\left.\left(\mathrm{R}_{\mathrm{b}}\right)\right]$.

\section{Results}

Inhibition of P450 Enzymes by Imetelstat in Cryopreserved Human Hepatocytes. Imetelstat $(0.1-100 \mu \mathrm{M})$ was evaluated as a potential direct inhibitor of $\mathrm{P} 450$ isoforms in $\mathrm{CHH}(0.5$ million cells $/ \mathrm{ml})$ at the approximate substrate $\mathrm{K}_{\mathrm{m}}$ concentration (see Table 1) as described in Materials and Methods. As shown in Fig. 2 and Table 2, imetelstat had little to no inhibitory effect on CYP1A2 (phenacetin), CYP2B6 (bupropion), CYP2C8 (amodiaquine), CYP2C9 (tolbutamide), CYP2C19 ( $S$ mephenytoin), CYP2D6 (dextromethorphan), CYP2E1 (chlorzoxazone), and CYP3A4 (midazolam) with $\mathrm{IC}_{50}$ values all $>100 \mu \mathrm{M}$. The maximum percent inhibition at the highest concentration of imetelstat tested $(100 \mu \mathrm{M})$ was less than $20 \%$ for all $\mathrm{P} 450$ isoforms, except for CYP2C8, where the maximum inhibition was $49 \%$. However, a modest inhibitory concentration dependency for CYP2C8 was observed, with inhibition reaching a plateau between 30 and $100 \mu \mathrm{M}$ imetelstat. In contrast, all positive control inhibitors showed $>80 \%$ inhibition of their respective $\mathrm{P} 450$ isoforms, as expected (see Table 2). Furthermore, a lack of CYP2C8 TDI by imetelstat was observed with an $\mathrm{IC}_{50}$ shift assay (Supplemental Fig. 1).

\section{Discussion}

For small-molecule drugs, regulatory agencies such as the FDA and EMA recommend in vitro evaluation of the drug-drug interaction (DDI) potential of a new molecular entity as part of the submission package toward drug approval. This is typically done through in vitro assessment of the inhibition of drug-metabolizing enzymes, namely P450 enzymes, in validated test-systems such as HLM or cryopreserved human hepatocytes $(\mathrm{CHH})$. In the case of oligonucleotide drugs, although these biomolecules as a class differ in structure and composition from conventional small-molecule drugs, assessment of the DDI potential of oligonucleotides is typically evaluated in a manner similar to the FDA and EMA DDI study recommendations outlined for small-molecule drugs. Thus, in the present study the in vitro potential for imetelstat to inhibit P450 enzymes, namely CYP1A2, CYP2B6, CYP2C8, CYP2C9, CYP2C19, CYP2D6, CYP2E1, and CYP3A4 was evaluated in CHH.

As shown in Fig. 2 and Table 2, imetelstat was found not to be an in vitro inhibitor of any $\mathrm{P} 450$ enzymes tested in $\mathrm{CHH}$ with $\mathrm{IC}_{50}$ values greater than $100 \mu \mathrm{M}$. Furthermore, the maximum percent inhibition at highest imetelstat concentration $(100 \mu \mathrm{M})$ was minimal $(<20 \%)$ for all P450 isoforms, except for CYP2C8 activity (49\% inhibition), where modest concentration dependency was observed. However, it is unclear why the inhibition of CYP2C8 plateaued between 30 and $100 \mu \mathrm{M}$ (45\%-49\% inhibition), as this is typically observed when inhibitor solubility is an issue; a phenomenon not observed with imetelstat (high aqueous solubility). Overall, these findings are consistent with other in vitro reports that oligonucleotides as a class do not cause significant inhibition of P450 enzymes in CHH (Shemesh et al., 2017; Kazmi et al., 
CYP1A2

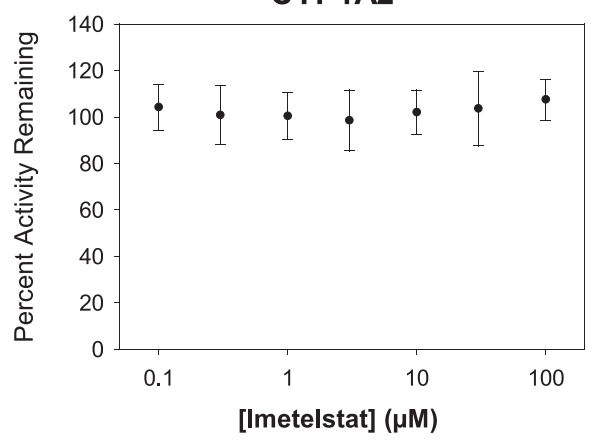

CYP2C8

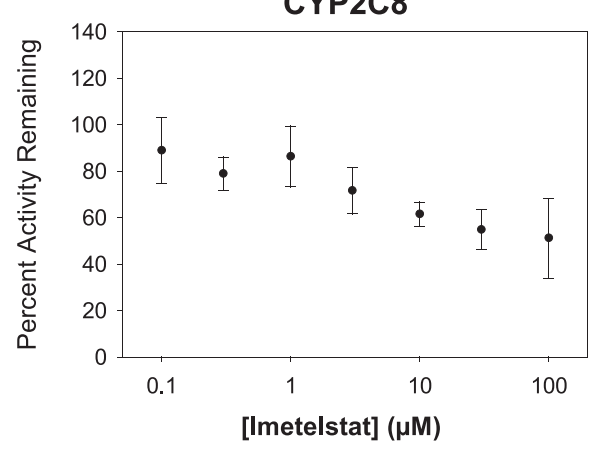

CYP2C19

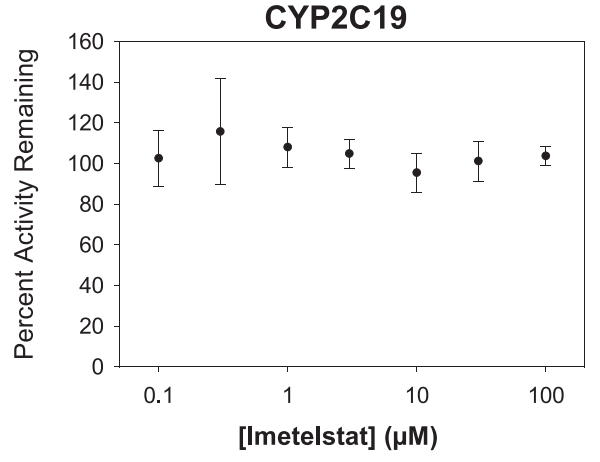

CYP2E1

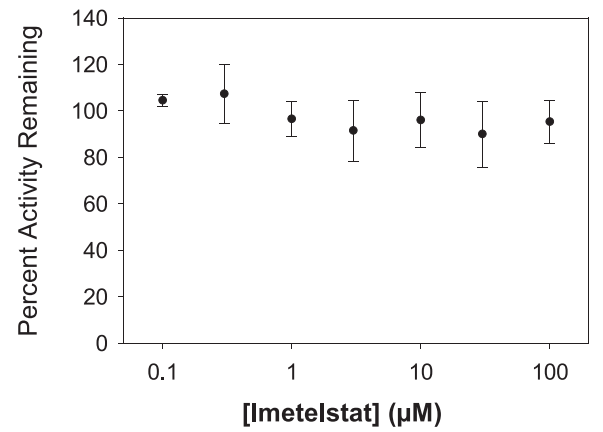

CYP2B6

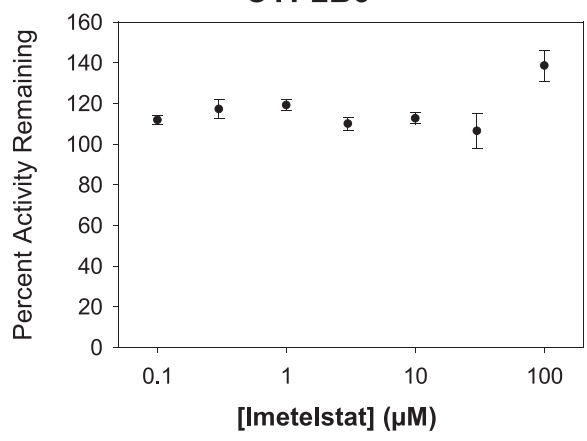

CYP2C9
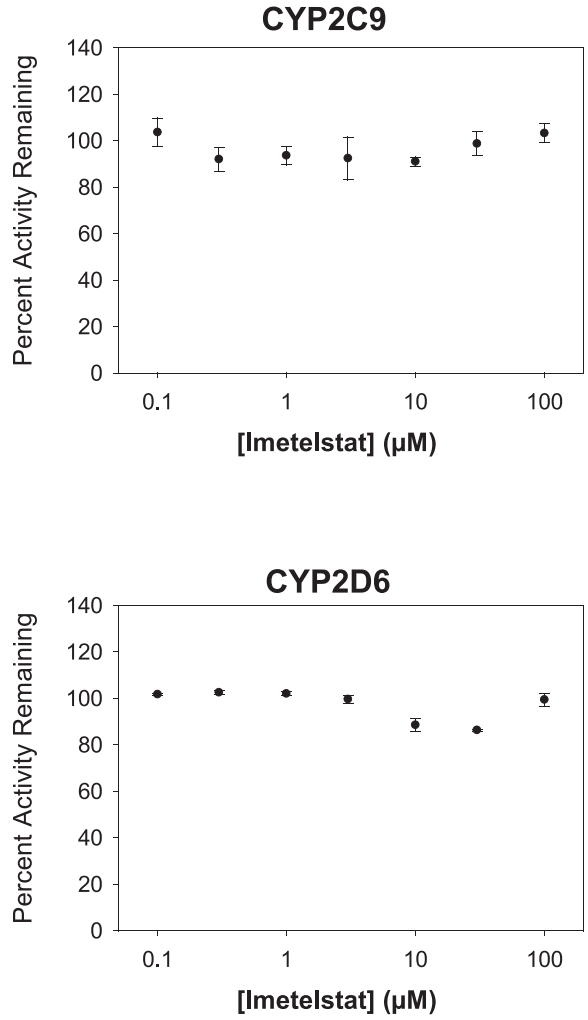

CYP3A4

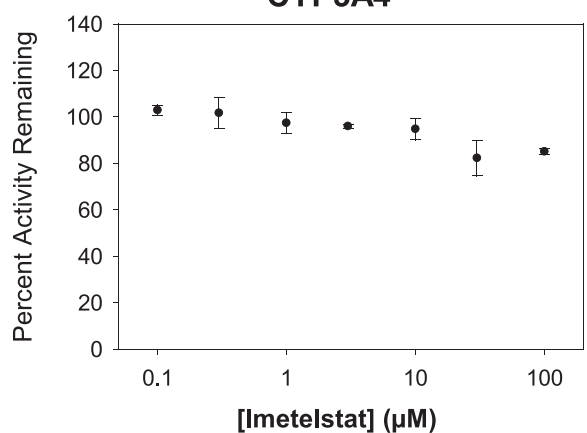

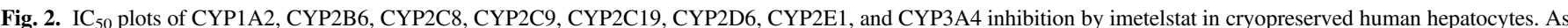

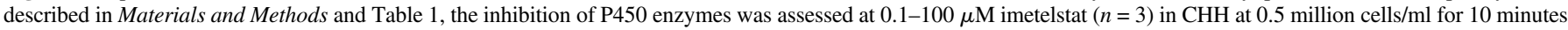
followed by liquid chromatography/tandem mass spectrometry analysis. Curve-fitting was not performed when $\mathrm{IC}_{50}$ values were projected to be $>100 \mu \mathrm{M}$. 
TABLE 2

$\mathrm{IC}_{50}$ and maximum percent inhibition values for the inhibition of P450 isoforms by imetelstat and positive control inhibitors in pooled cryopreserved human hepatocytes

\begin{tabular}{|c|c|c|c|c|c|}
\hline Enzyme & Substrate & $\mathrm{IC}_{50}$ & Maximum Inhibition & $\begin{array}{l}\text { Positive Control Inhibitor } \\
\text { (Concentration) }\end{array}$ & Positive Control Inhibition \\
\hline & & $\mu M$ & $\%$ & & $\%$ \\
\hline CYP1A2 & Phenacetin & $>100$ & $<10$ & $\alpha$-Naphthoflavone $(1 \mu \mathrm{M})$ & 90 \\
\hline CYP2B6 & Bupropion & $>100$ & $<10$ & Ticlopidine $(10 \mu \mathrm{M})$ & 84 \\
\hline CYP2C8 & Amodiaquine & $>100$ & 49 & Montelukast $(50 \mu \mathrm{M})$ & 88 \\
\hline CYP2C9 & Tolbutamide & $>100$ & $<10$ & Sulfaphenazole $(5 \mu \mathrm{M})$ & 99 \\
\hline CYP2C19 & $S$-Mephenytoin & $>100$ & $<10$ & $N$-3-Benzylphenobarbital $(4 \mu \mathrm{M})$ & 96 \\
\hline CYP2D6 & Dextromethorphan & $>100$ & 14 & Quinidine $(5 \mu \mathrm{M})$ & 99 \\
\hline CYP2E1 & Chlorzoxazone & $>100$ & 10 & 4-Methylpyrazole $(10 \mu \mathrm{M})$ & 92 \\
\hline CYP3A4 & Midazolam & $>100$ & 18 & Ketoconazole $(4 \mu \mathrm{M})$ & 98 \\
\hline
\end{tabular}

2018). The recent report by Kazmi and colleagues (2018) suggests that oligonucleotide molecules as a class may cause differential testsystem-dependent inhibition in HLM and CHH based on the chemical properties of the oligonucleotide backbone (phosphodiester vs. phosphorothioate linkage), and that inhibition profiles for oligonucleotides in $\mathrm{CHH}$ are more clinically relevant. In the case of imetelstat, a thio-phosphoramidate linkage is present (see Fig. 1) along with a 5 '-palmitoyl chain for the improvement of biologic stability and tissue uptake (Herbert et al., 2005). Liver uptake of imetelstat has been previously observed in mice (Herbert et al., 2005), consistent with in house preclinical toxicology studies where liver distribution of imetelstat was seen in rodents and primates, confined largely to Kupffer cells (data not shown). This accumulation into tissue macrophages (often observed as basophilic granules) has been shown to be a class effect of oligonucleotides, especially with high doses (Frazier, 2015). In screening studies, potent $\mathrm{P} 450$ inhibition by imetelstat was observed in HLM for CYP2C8 ( $\mathrm{IC}_{50}$ value of $0.7 \mu \mathrm{M}$ ) with $\mathrm{IC}_{50}$ values for other P450 enzymes spanning 1.8-26 $\mu \mathrm{M}$ (see Supplemental Table 1). The P450 inhibition observed in HLM, particularly of CYP2C8, was completely attenuated in the present study conducted with $\mathrm{CHH}$, suggesting test-system-dependent inhibition, possibly due to the thiophosphoramidate linkage of imetelstat.

With respect to predictions of a clinical DDI, the static basic model approach, as recommended by the EMA and FDA, was first used where $\mathrm{R}_{1}=1+\mathrm{I}_{\text {max, } \mathrm{u}} / \mathrm{K}_{\mathrm{i}}$, with $\mathrm{R}_{1}$ representing the predicted area under the curve (AUC) ratio in the presence or absence of inhibitor, $I_{\max , u}$ representing maximal free plasma concentration of the inhibitor, and $\mathrm{K}_{\mathrm{i}}$ representing the unbound in vitro inhibition constant. Imetelstat $\mathrm{C}_{\max }$ concentration with a 2-hour intravenous infusion dosage of $9.4 \mathrm{mg} / \mathrm{kg}$ was approximately $30 \mu \mathrm{M}(136 \mu \mathrm{g} / \mathrm{ml})$. The in vitro $\mathrm{K}_{\mathrm{i}}$ value for $\mathrm{P} 450$ inhibition can be predicted reliably from the $\mathrm{IC}_{50}$ value by using the equation $\mathrm{IC}_{50} / 2=\mathrm{K}_{\mathrm{i}}$ (Haupt et al., 2015). Nonspecific binding of imetelstat to $\mathrm{CHH}$ was expected to be negligible $\left(\mathrm{fu}_{\mathrm{inc}}=1\right)$ based on physicochemical properties (hydrophilicity [predicted $\log \mathrm{P}=-3.19$ ] and charge status), and application of the predicted hepatocyte binding equation described by Kilford et al. (2008). In the case of CYP2C8 (the $\mathrm{P} 450$ enzyme with the greatest maximal inhibition by imetelstat), the $\mathrm{K}_{\mathrm{i}}$ was estimated to be $50 \mu \mathrm{M}$ based on an approximate $\mathrm{IC}_{50}$ value of $100 \mu \mathrm{M}$ in $\mathrm{CHH}$. Considering the plasma protein binding of imetelstat (at least $92.7 \%$; Janssen internal data), the $\mathrm{R}_{1}$ value was calculated as 1.04 , which is above the EMA and FDA cutoff criteria of $R_{1} \geq 1.02$. The basic static models recommended by the EMA and FDA are designed to be stringent in nature by including a 50-fold safety margin for $\mathrm{I} / \mathrm{K}_{\mathrm{i}}(\mathrm{I}=1$, $K_{i}=50$; or $\left.I / K_{i}=0.02\right)$. Should an assessment with the basic static model fail to pass DDI cutoff criteria, the EMA and FDA recommend assessment of DDI prediction with a static mechanistic model or a dynamic model, which incorporate much more detailed drug disposition parameters than the basic static model (Fahmi et al., 2009). For the static mechanistic model, these parameters include the effect of hepatic reversible inhibition, time-dependent inhibition (TDI), enzyme induction, and hepatic inlet concentration of a perpetrator drug; as well as fractional metabolism $\left(f_{m}\right)$ of a victim drug. However, the EMA and FDA recommend, in their most recent DDI guidelines, that inhibitory potential and induction potential with the static mechanistic model be considered separately and not simultaneously, as was originally proposed by Fahmi and colleagues (2009). Thus, predictions of inhibitory perpetrator DDI potential with this model are to be based on an assessment of direct and time-dependent inhibition only (as shown in eq. 2). Furthermore, use of the static mechanistic approach described by the EMA and FDA in their respective DDI guidances requires an estimate of hepatic inlet concentration $\left(I_{h}\right)$ that is obtained by including absorption, dose, blood flow, and blood-partitioning parameters, in addition to plasma $C_{\max }$ and plasma protein-binding parameters (Ito et al., 1998). However, in the case of imetelstat, because it is delivered intravenously, oral absorption parameters are not applicable and the hepatic inlet concentration is equivalent to the unbound systemic plasma concentration ( $\mathrm{fu}_{\text {plasma }} \mathrm{XC}_{\max }=\mathrm{I}_{\mathrm{h}}=2.19 \mu \mathrm{M}$ ). Imetelstat is not expected to be a time-dependent inhibitor of CYP2C8 (Supplemental Fig. 1), and assuming a worst-case scenario of a victim drug with $100 \%$ systemic clearance through one P450 pathway $\left(f_{m}=1\right)$, application of these parameters to the static mechanistic model yields a prediction of 1.04fold change in the AUC of a victim drug that is a substrate of CYP2C8. This is well below the EMA and FDA AUC ratio cutoff criteria of $\geq 1.25$ and indicates that imetelstat would likely not be a perpetrator of a clinical DDI.

The predicted lack of clinical DDI for imetelstat is consistent with the reported lack of clinical interactions with other oligonucleotides. Clinical pharmacokinetic interaction studies with simvastatin (CYP3A4-mediated clearance), warfarin (CYP2C9/3A4/1A2-mediated clearance), rosiglitazone (CYP2C8/2C9-mediated clearance), glipizide (CYP2C9/2C8-mediated clearance), ezetimibe (glucuronidation-mediated clearance), metformin (renal transporter-mediated clearance), cisplatin (renal and copper transporter-mediated clearance), and gemcitabine (nucleoside kinasemediated clearance) have all shown no significant pharmacokinetic interaction with therapeutic oligonucleotides (Adjei et al., 2003; Villalona-Calero et al., 2004; Geary et al., 2006; Yu et al., 2009; Li et al., 2014). Furthermore, an in vivo rat preclinical interaction study with imetelstat coadministered with paclitaxel, which is metabolized by CYP3A1/2 in rat (Vaclavikova et al., 2004), showed no significant pharmacokinetic interaction (Janssen internal data), supporting the lack of reported DDIs by oligonucleotide-based therapeutics.

In conclusion, the findings presented in this study indicate that imetelstat is not a significant in vitro inhibitor of P450 enzymes in $\mathrm{CHH}$, and the in vitro-to-in vivo extrapolation (IVIVE) of DDI 
potential using the EMA- and FDA-recommended static mechanistic model also projects that imetelstat is not likely to be a clinically significant P450 inhibitor, and thus consequently unlikely to affect the pharmacokinetics of concomitant pharmacotherapies that are metabolized by P450s.

\section{Acknowledgments}

Medical writing assistance was provided by Ashwini Patil (Tata Consultancy Services, Mumbai, India) and additional editorial support was provided by Namit Ghildyal (Janssen Research \& Development, LLC). We would like to thank Shannon Dallas and Avijit Ghosh for helpful discussions and manuscript review.

\section{Authorship Contributions}

Participated in research design: Kazmi, Sensenhauser, Greway.

Conducted experiments: Kazmi, Sensenhauser.

Performed data analysis: Kazmi, Sensenhauser.

Wrote or contributed to the writing of the manuscript: Kazmi, Sensenhauser, Greway.

\section{References}

Aartsma-Rus A (2016) New momentum for the field of oligonucleotide therapeutics. Mol Ther $\mathbf{2 4}$ 193-194.

Adjei AA, Dy GK, Erlichman C, Reid JM, Sloan JA, Pitot HC, Alberts SR, Goldberg RM, Hanson LJ, Atherton PJ, et al. (2003) A phase I trial of ISIS 2503, an antisense inhibitor of H-ras, in combination with gemcitabine in patients with advanced cancer. Clin Cancer Res 9:115-123.

Baerlocher GM, Oppliger Leibundgut E, Ottmann OG, Spitzer G, Odenike O, McDevitt MA, Röth A Daskalakis M, Burington B, Stuart M, et al. (2015) Telomerase inhibitor imetelstat in patients with essential thrombocythemia. $N$ Engl J Med 373:920-928.

Chen C, Yang Z, and Tang X (2018) Chemical modifications of nucleic acid drugs and their delivery systems for gene-based therapy. Med Res Rev 38:829-869.

Chiappori AA, Kolevska T, Spigel DR, Hager S, Rarick M, Gadgeel S, Blais N, Von Pawel J, Hart L, Reck M, et al. (2015) A randomized phase II study of the telomerase inhibitor imetelstat as maintenance therapy for advanced non-small-cell lung cancer. Ann Oncol 26:354-362.

Deleavey GF and Damha MJ (2012) Designing chemically modified oligonucleotides for targeted gene silencing. Chem Biol 19:937-954.

Dillen L, Sips L, Greway T, and Verhaeghe T (2017) Quantitative analysis of imetelstat in plasma with LC-MS/MS using solid-phase or hybridization extraction. Bioanalysis 9:1859-1872.

Fahmi OA, Hurst S, Plowchalk D, Cook J, Guo F, Youdim K, Dickins M, Phipps A, Darekar A, Hyland R, et al. (2009) Comparison of different algorithms for predicting clinical drug-drug interactions, based on the use of CYP3A4 in vitro data: predictions of compounds as precipitants of interaction. Drug Metab Dispos 37:1658-1666.

Frazier KS (2015) Antisense oligonucleotide therapies: the promise and the challenges from a toxicologic pathologist's perspective. Toxicol Pathol 43:78-89.

Geary RS, Bradley JD, Watanabe T, Kwon Y, Wedel M, van Lier JJ, and VanVliet AA (2006) Lack of pharmacokinetic interaction for ISIS 113715, a 2'-0-methoxyethyl modified antisense oligonucleotide targeting protein tyrosine phosphatase 1B messenger RNA, with oral antidiabetic compounds metformin, glipizide or rosiglitazone. Clin Pharmacokinet 45:789-801.

Haupt LJ, Kazmi F, Ogilvie BW, Buckley DB, Smith BD, Leatherman S, Paris B, Parkinson O, and Parkinson A (2015) The reliability of estimating Ki values for direct, reversible inhibition of cytochrome P450 enzymes from corresponding IC50 values: a retrospective analysis of 343 experiments. Drug Metab Dispos 43:1744-1750.

Herbert BS, Gellert GC, Hochreiter A, Pongracz K, Wright WE, Zielinska D, Chin AC, Harley CB, Shay JW, and Gryaznov SM (2005) Lipid modification of GRN163, an N3'-->P5' thiophosphoramidate oligonucleotide, enhances the potency of telomerase inhibition. Oncogene 24 $5262-5268$.

Ito K, Iwatsubo T, Kanamitsu S, Ueda K, Suzuki H, and Sugiyama Y (1998) Prediction of pharmacokinetic alterations caused by drug-drug interactions: metabolic interaction in the liver. Pharmacol Rev 50:387-412.

Kazmi F, Yerino P, McCoy C, Parkinson A, Buckley DB, and Ogilvie BW (2018) An assessment of the in vitro inhibition of cytochrome P450 enzymes, UDP-glucuronosyltransferases, and transporters by phosphodiester- or phosphorothioate-linked oligonucleotides. Drug Metab Dispos 46:1066-1074.

Kilford PJ, Gertz M, Houston JB, and Galetin A (2008) Hepatocellular binding of drugs: correction for unbound fraction in hepatocyte incubations using microsomal binding or drug lipophilicity data. Drug Metab Dispos 36:1194-1197.

Li Z, Hard ML, Grundy JS, Singh T, von Moltke LL, and Boltje I (2014) Lack of clinical pharmacodynamic and pharmacokinetic drug-drug interactions between warfarin and the antisense oligonucleotide mipomersen. J Cardiovasc Pharmacol 64:164-171.

Lichtsteiner SP, Lebkowski JS, and Vasserot AP (1999) Telomerase. A target for anticancer therapy. Ann N Y Acad Sci 886:1-11.

Lundin KE, Gissberg O, and Smith CI (2015) Oligonucleotide therapies: the past and the present. Hum Gene Ther 26:475-485.

Marian CO, Cho SK, McEllin BM, Maher EA, Hatanpaa KJ, Madden CJ, Mickey BE, Wright WE, Shay JW, and Bachoo RM (2010) The telomerase antagonist, imetelstat, efficiently targets glioblastoma tumor-initiating cells leading to decreased proliferation and tumor growth. Clin Cancer Res 16:154-163.

Prakash TP (2011) An overview of sugar-modified oligonucleotides for antisense therapeutics. Chem Biodivers 8:1616-1641.

Röth A, Harley CB, and Baerlocher GM (2010) Imetelstat (GRN163L)--telomerase-based cance therapy. Recent Results Cancer Res 184:221-234.

Sharma VK and Watts JK (2015) Oligonucleotide therapeutics: chemistry, delivery and clinica progress. Future Med Chem 7:2221-2242.

Shemesh CS, Yu RZ, Warren MS, Liu M, Jahic M, Nichols B, Post N, Lin S, Norris DA, Hurh E, et al. (2017) Assessment of the drug interaction potential of unconjugated and $\mathrm{GalNAc}_{3}$-conjugated 2'-MOE-ASOs. Mol Ther Nucleic Acids 9:34-47.

Stein CA and Castanotto D (2017) FDA-approved oligonucleotide therapies in 2017. Mol Ther 25: 1069-1075.

Tefferi A, Lasho TL, Begna KH, Patnaik MM, Zblewski DL, Finke CM, Laborde RR, Wassie E, Schimek L, Hanson CA, et al. (2015) A pilot study of the telomerase inhibitor imetelstat for myelofibrosis. $N$ Engl J Med 373:908-919.

Vaclavikova R, Soucek P, Svobodova L, Anzenbacher P, Simek P, Guengerich FP, and Gut (2004) Different in vitro metabolism of paclitaxel and docetaxel in humans, rats, pigs, and minipigs. Drug Metab Dispos 32:666-674.

Villalona-Calero MA, Ritch P, Figueroa JA, Otterson GA, Belt R, Dow E, George S, Leonardo J, McCachren S, Miller GL, et al. (2004) A phase I/II study of LY900003, an antisense inhibitor of protein kinase $\mathrm{C}$-alpha, in combination with cisplatin and gemcitabine in patients with advanced non-small cell lung cancer. Clin Cancer Res 10:6086-6093.

Yu RZ, Geary RS, Flaim JD, Riley GC, Tribble DL, vanVliet AA, and Wedel MK (2009) Lack of pharmacokinetic interaction of mipomersen sodium (ISIS 301012), a 2'-O-methoxyethyl modified antisense oligonucleotide targeting apolipoprotein B-100 messenger RNA, with simvastatin and ezetimibe. Clin Pharmacokinet 48:39-50.

Address correspondence to: Dr. Faraz Kazmi, Janssen Research and Development, 1400 McKean Road, Spring House, PA 19477. E-mail: fkazmi@its.jnj.com 